\title{
The Aberdeen Weight-Bearing Test (Knee): a new objective test for anterior knee discomfort
}

\author{
David Robert Walker MacDonald ${ }^{1} \cdot$ Haroon Rehman ${ }^{1} \cdot$ Carol Ann Carnegie $^{1} \cdot$ Jordi Tomas-Hernandez $^{2}$. \\ Alan John Johnstone ${ }^{1}$
}

Received: 4 January 2018 / Accepted: 17 July 2018 / Published online: 20 July 2018

(c) The Author(s) 2018

\begin{abstract}
Purpose We present the Aberdeen Weight-Bearing Test (Knee), an objective test specific for anterior knee discomfort assessed via load bearing. We assess its validity by performing it on normal subjects with no knee symptoms and subjects who had undergone anterograde tibial nailing.

Methods Two scales are placed parallel on the floor with the dials concealed from the subject. The subject then kneels with one knee on each scale. The weight through each knee is recorded at $0,15,30,45$, and $60 \mathrm{~s}$. The proportion of total body weight on each leg at each timepoint is calculated, and a ratio calculated from the values. A value of 1 equates to equal weight on each leg. The test was performed on 53 normal subjects and 38 subjects who had undergone tibial nailing.

Results In the normal group, no significant difference in mean ratio of weight distribution (left:right) was seen at any timepoint (mean ratio range $=0.98-0.99, p$ value range $=0.18-0.64$ ). In the tibial nail group, a difference was observed in mean ratio of weight distribution (injured:uninjured) favouring the uninjured leg, reaching significance at $0,15,30$, and $45 \mathrm{~s}$ (mean ratio range $=0.88-0.94, p$ value range $=0.01-0.02)$. At $60 \mathrm{~s}$, the mean ratio was $0.93(p=0.09)$.

Conclusion The Aberdeen Weight-Bearing Test (Knee) is an objective, easily reproducible, specific test for anterior knee discomfort. It produces different results in individuals who have undergone anterograde tibial nailing compared to individuals with no knee symptoms.
\end{abstract}

Keywords Outcome measure $\cdot$ Clinical assessment $\cdot$ Knee $\cdot$ Anterior knee pain $\cdot$ Tibial nail

\section{Introduction}

Anterior knee discomfort following anterograde intramedullary nailing of the tibia is a widely recognised complication which can cause significant morbidity and disability [1]. It occurs commonly with incidences between 8.7 and $37 \%$ reported $[2,3]$. There is increasing interest in the literature in the use of the suprapatellar approach rather than the traditional infrapatellar approach as this may reduce this complication. Although to our knowledge, no randomised controlled trial has been published, other studies comparing these approaches have used subjective patient-reported

David Robert Walker MacDonald

david.macdonald7@nhs.net

1 Aberdeen Royal Infirmary, Foresterhill, Aberdeen AB25 2ZN, UK

2 Vall d'Hebron Hospital, Barcelona, Spain outcome measures (PROMs) rather than objective outcome measures $[4,5]$.

The PROMs used such as the Oxford knee score and Lysholm knee score are not specific for anterior knee pain, but rather reflect overall knee function [6, 7]. Whilst PROMs such as the Kujala score [8] and Fulkerson's modification of the Lysholm score [9] are more specific for anterior knee pain, they suffer from the same disadvantages as other PROMs.

PROMs are frequently used as outcome measures as they reflect a patient's self-perceived burden of disease, which is important in clinical practice. They are readily available and can be posted to patients or completed online. However, they have significant drawbacks. These include "floor" and "ceiling" effects [10] such as the preoperative floor effects and postoperative ceiling effects seen in the WOMAC and SF-36 scores of patients undergoing total hip arthroplasty [11], and the ceiling effects seen in the Harris hip scores of patients undergoing total 
hip arthroplasty [12]. PROMs can also give mixed messages and can be negatively influenced by psychosocial factors $[13,14]$, measuring the patient's perception of an outcome, rather than the true outcome.

To adequately assess the impact of the suprapatellar approach compared to the infrapatellar approach on anterior knee pain, it is necessary to use objective outcome measures in addition to PROMs. Detailed measurement of joint motion and muscle activity using equipment such as pedometers and accelerometers can be a useful objective outcome measure. However, this requires specialist equipment and training only available in certain centres. These problems can be addressed using objective performance-based functional outcome tools in which the patient is observed performing tasks such as walking, rising from a chair or climbing stairs, and their performance quantified in time, counting or distance [15]. Examples include the Timed Up and Go Test [16], 6-Minute Walk Test [17] and Stair Climbing Test [18]. These do not correlate well with PROMs suggesting that PROMs may not fully reflect functional performance [15]. Functional outcome tools are often also not specific to site and pathology.

We present The Aberdeen Weight-Bearing Test (Knee) (AWT-K), an objective test specific for anterior knee discomfort assessed via direct load bearing. We assessed its validity by performing it on subjects with no knee symptoms and subjects who had undergone anterograde tibial nailing. It may be a useful objective outcome measure to quantify anterior knee discomfort following anterograde tibial nailing.

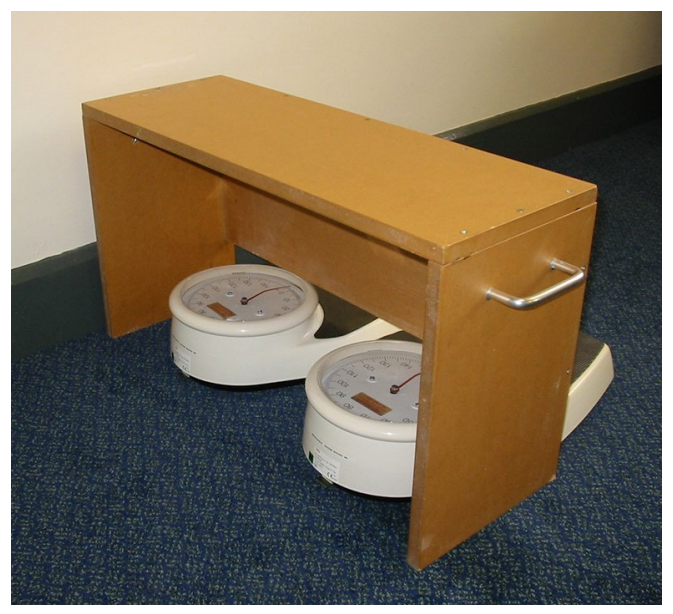

Fig. 1 Two scales are placed on the floor with dials concealed from the subject

\section{Methods}

\section{Aberdeen Weight-Bearing Test (Knee)}

Two equally calibrated scales are placed in a parallel configuration on the floor. The dials are concealed from the test subject by a box (Figs. 1, 2).

Patient weight is calculated as the sum of the scale readings as the subject stands with one foot on each scale. The subject then kneels with one knee on each scale. The anterior thighs should be gently pressed against the box to maximise weight through the knee (Fig. 3). The feet may touch the ground behind the scales to maintain balance, but should bear minimum weight.

The weight passing through each knee is then recorded at $0,15,30,45$, and $60 \mathrm{~s}$. The test ends at $60 \mathrm{~s}$, or if the subject is unable to continue the test for the full $60 \mathrm{~s}$.

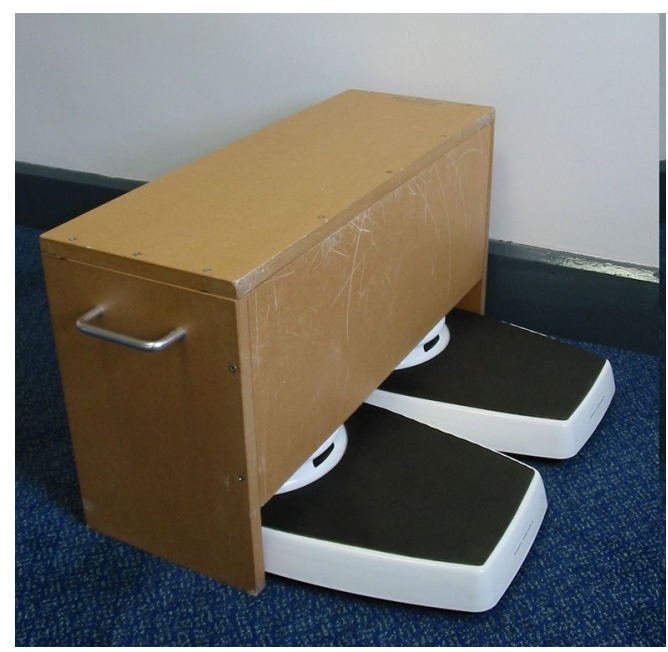

Fig. 2 Two scales are placed on the floor with dials concealed from the subject

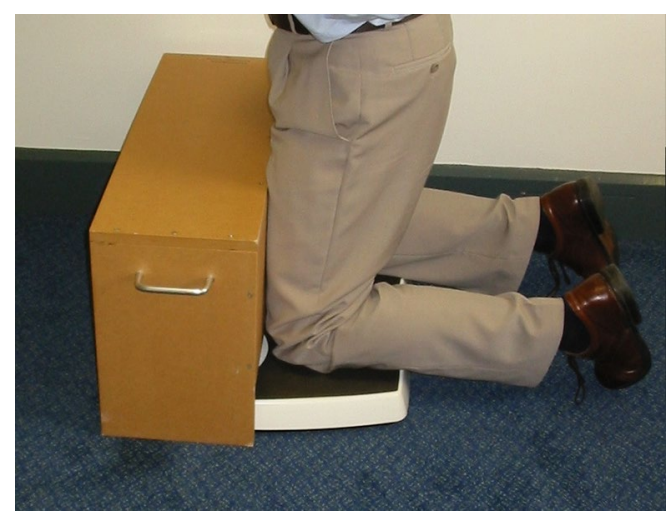

Fig. 3 Subject kneels with one knee on each scale 
To account for differences in total weight between subjects, the weight on each knee is converted to a proportion of the subject's total body weight. A ratio is calculated from the values (injured knee weight/total weight on scales):(uninjured knee weight/total weight on scales), with a value of 1 representing equal weight on each knee. In our study in subjects who had sustained an injury, the injured leg was compared to the uninjured leg. In uninjured patients, the left leg was compared to the right.

\section{Study design}

To determine whether the Aberdeen Weight-Bearing Test (Knee) produced different results in subjects with lower limb injury and uninjured subjects, the test was performed on 104 individuals. The normal population was represented by 53 subjects with no history lower limb injury or pathology. The injured population was represented by 51 subjects who had undergone anterograde intramedullary tibial nailing using either a suprapatellar (33) or infrapatellar (18) approach (Fig. 4). The test was performed at a mean of 27 months after surgery (range 6-52 months, $\mathrm{SD}=14$ ). The individuals also completed Fulkerson [9] and Irrgang's scores [19] (commonly used PROMs assessing knee function) at the same review point and these were correlated with their weight-bearing test results. Prior to data collection, our scales were calibrated using standard $10 \mathrm{~kg}$ weights.

The Statistical Package for the Social Science SPSS v20 (SPSS Inc, Chicago, IL, USA) was used to analyse the data. A one-tail $t$ test with a test value of 0 was used to assess weight differences between limbs in injured and uninjured patients. A one-tail $t$ test with a test value of 1 was used to assess ratio of weight distribution in injured and uninjured patients. The spearman's rank correlation test was used to assess the relationship between AWT-K, Fulkerson, and Irrgang scores.
Fig. 4 Injured and uninjured groups

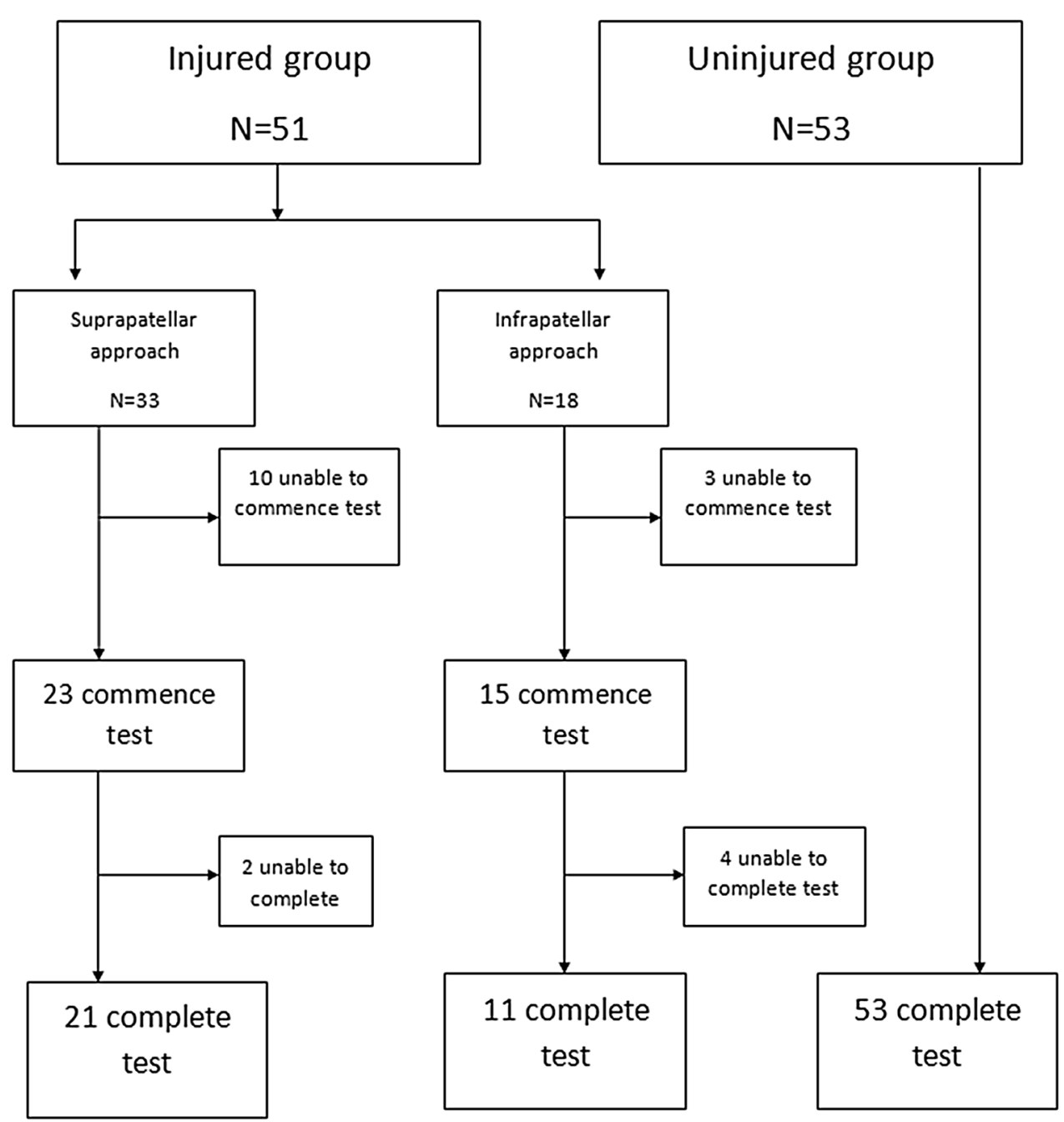


Table 1 Demographic data for injured and uninjured groups

\begin{tabular}{llllll}
\hline & Sample size & Age & Male & Female & Weight \\
\hline Normal & 53 & $42 \pm 13(22-60)$ & $26(49 \%)$ & $27(51 \%)$ & $77.6 \pm 15.4(48-120)$ \\
Injured & 51 & $42 \pm 13(22-68)$ & $37(73 \%)$ & $14(27 \%)$ & $84.1 \pm 18.7(60-170)$ \\
\hline
\end{tabular}

Table 2 Mean difference in weight distribution for normal group

\begin{tabular}{lllll}
\hline & $N$ & $\begin{array}{l}\text { Mean difference weight distri- } \\
\text { bution left-right }(\mathrm{kg})\end{array}$ & SD & Sig. \\
\hline $0 \mathrm{~s}$ & 53 & -0.1 & 3.9 & 0.33 \\
$15 \mathrm{~s}$ & 53 & -0.9 & 4.0 & 0.11 \\
$30 \mathrm{~s}$ & 53 & -0.9 & 4.0 & 0.11 \\
$45 \mathrm{~s}$ & 53 & 0 & 3.8 & 0.37 \\
$60 \mathrm{~s}$ & 53 & -1.1 & 4.3 & 0.08 \\
\hline
\end{tabular}

Table 3 Mean ratio of weight distribution for normal group

\begin{tabular}{lllll}
\hline & $N$ & $\begin{array}{l}\text { Mean ratio weight distri- } \\
\text { bution left:right }\end{array}$ & SD & Sig. \\
\hline $0 \mathrm{~s}$ & 53 & 0.99 & 0.11 & 0.64 \\
$15 \mathrm{~s}$ & 53 & 0.98 & 0.11 & 0.22 \\
$30 \mathrm{~s}$ & 53 & 0.98 & 0.11 & 0.21 \\
$45 \mathrm{~s}$ & 53 & 0.99 & 0.11 & 0.62 \\
$60 \mathrm{~s}$ & 53 & 0.98 & 0.12 & 0.18 \\
\hline
\end{tabular}

\section{Results}

A total of 104 subjects were recruited. The normal population was represented by 53 subjects. 51 patients that had undergone intramedullary nail fixation for tibial shaft fractures were tested. A suprapatellar approach was used in 33 patients and an infrapatellar approach in 18 patients. Of the 51 injured patients, 13 were unable to commence the kneeling test due to pain and were excluded. Of the remaining 38,6 were unable to complete the test due to pain (Fig. 4). Demographic data is provided in Table 1.

\section{Normal population}

No significant difference in weight distribution $(\mathrm{kg})$ across the knees was detectable for our test population at any time interval (Table 2). Similarly, there was no significant difference in the ratio of weight distributed through both limbs (left:right) (Table 3).
Table 4 Mean difference in weight distribution for injured group

\begin{tabular}{lllrl}
\hline & $N$ & $\begin{array}{l}\text { Mean difference weight distri- } \\
\text { bution injured-uninjured }\end{array}$ & SD & Sig. \\
\hline $0 \mathrm{~s}$ & 38 & 4.7 & 11.7 & 0.03 \\
$15 \mathrm{~s}$ & 37 & 5.5 & 9.1 & 0.001 \\
$30 \mathrm{~s}$ & 36 & 4.0 & 9.3 & 0.01 \\
$45 \mathrm{~s}$ & 35 & 4.6 & 9.4 & 0.01 \\
$60 \mathrm{~s}$ & 32 & 3.1 & 8.4 & 0.05 \\
\hline
\end{tabular}

Table 5 Mean ratio of weight distribution for injured group

\begin{tabular}{lllll}
\hline & $N$ & $\begin{array}{l}\text { Mean ratio weight distribu- } \\
\text { tion injured:uninjured }\end{array}$ & SD & Sig. \\
\hline $0 \mathrm{~s}$ & 38 & 0.91 & 0.25 & 0.03 \\
$15 \mathrm{~s}$ & 37 & 0.88 & 0.22 & 0.002 \\
$30 \mathrm{~s}$ & 36 & 0.92 & 0.23 & 0.04 \\
$45 \mathrm{~s}$ & 35 & 0.91 & 0.22 & 0.02 \\
$60 \mathrm{~s}$ & 32 & 0.94 & 0.20 & 0.09 \\
\hline
\end{tabular}

Table 6 Correlation between AWT-K, Fulkerson, and Irrgang scores

\begin{tabular}{lll}
\hline & \multicolumn{2}{l}{ Spearman's correlation coefficient } \\
\cline { 2 - 3 } & Fulkerson score & Irrgang score \\
\hline AWT-K 0 s & 0.418 & 0.247 \\
$N=38$ & $p=0.01$ & $p=0.14$ \\
AWT-K 15 s & 0.326 & 0.157 \\
$N=37$ & $p=0.05$ & $p=0.36$ \\
AWT-K 30 s & 0.353 & 0.147 \\
$N=36$ & $p=0.04$ & $p=0.39$ \\
AWT-K 45 s & 0.308 & 0.089 \\
$N=35$ & $p=0.07$ & $p=0.61$ \\
AWT-K 60 s & 0.336 & 0.196 \\
$N=32$ & $p=0.06$ & $p=0.28$ \\
Fulkerson score & 1.000 & 0.862 \\
$N=38$ & & $p<0.001$ \\
Irrgang score & 0.862 & 1.000 \\
$N=38$ & $p<0.001$ & \\
\hline
\end{tabular}

\section{Injured population}

A significant difference in weight distributed across the knees was observed at all timepoints (Table 4). A significant difference in the ratio of weight distributed across each knee (as a proportion of total weight) was observed at 
$0,15,30$, and $45 \mathrm{~s}$, and there was greater variability in the ratio of weight tolerated through injured limbs (Table 5).

Although there was a trend toward decreasing correlation with the Fulkerson score as time progressed, no statistical significance was noted. A statistically significant correlation was observed between the AWT-K at $45 \mathrm{~s}$ and the Irrgang score $(p<0.05)$ (Table 6). A statistically significant correlation was noted between the Irrgang and Fulkerson scores.

\section{Discussion}

We believe that the Aberdeen Weight-Bearing Test (Knee) is an easily reproducible outcome measure for anterior knee discomfort assessed via direct load tolerance. Unlike other outcome measures, it is objective, and specific to anterior knee discomfort. It does not require specialist equipment or training, and can be easily performed in an outpatient setting. Our results demonstrate that the test produces different results in individuals who have been treated using an anterograde tibial nail, compared to normal individuals.

The differences in AWT-K result were significant at all but one timepoint in injured patients. It is likely that significance was not reached at $60 \mathrm{~s}$ due to the limited number of injured subjects in our study. It seems likely that with further recruitment, the difference found will reach significance.

A potential weakness of the test is the possibility that a subject could unevenly shift his weight onto his feet during the test, altering the test result. However, our data show a consistency in mean proportion of total body weight on the knees compared to the feet across all timepoints in both groups. In the uninjured group, the mean proportion of total body weight through the scales compared to the feet ranged from 0.96 to 0.97 , whilst in the injured group, it ranged from 0.90 to 0.91 . Another potential weakness is that 13 of the 51 injured patients were unable to commence the weight-bearing test due to pain, and a further six patients were unable to complete the test due to pain. However, this itself can be used as part of the outcome measure, and is a useful indication of level of function. A further weakness of the study is that the AWT-K was performed at variable time period following surgery in the injured group, and this is likely to have influenced results. This does not alter our conclusion that the AWT-K produces different results in patients who have undergone anterograde tibial nailing compared to normal individuals, but if used in future studies the, the AWT-K will be more meaningful if used at regimented timepoints.

Our results show a poor correlation between the AWT-K and the subjective Fulkerson and Irrgang tests. The poor correlation may indicate a poor correlation between subjective patient-reported symptoms and the objective measurements, as has previously been shown [20]. It may also have been influenced by the heterogeneity of our injured sample group. Some had their surgery performed via an infrapatellar approach and some via a suprapatellar approach that may give a lower incidence of anterior knee discomfort. We plan to perform subgroup analysis to assess this possibly following further patient recruitment to this study.

Although PROMs have value in determining patients' self-perceived burden of disease, they are affected by psychosocial factors and many are not specific. By contrast, the AWT-K is an objective outcome measure for anterior knee discomfort assessed through load bearing. We have demonstrated its use as an outcome measure for anterior knee discomfort following anterograde tibial nailing. In the future, it may be used as an outcome measure in other procedures or conditions known to produce anterior knee pain such as anterior cruciate ligament reconstruction or chondromalacia patellae. It may also be easily modified to be used as an outcome measure for other lower limb procedures or conditions.

\section{Conclusion}

We present the Aberdeen Weight-Bearing Test (Knee); an objective test specific for anterior knee discomfort assessed via load bearing. We conclude that the AWT-K produces different results in individuals who have undergone anterograde tibial nailing compared to normal individuals. It may be a useful, objective outcome measure following anterograde tibial nailing, as well as other procedures and conditions.

\section{Compliance with ethical standards}

Conflict of interest David R. W. MacDonald, Haroon Rehman, Carol Carnegie, Jordi Tomas-Hernadez, and Alan J. Johnstone declare that they have no conflict of interest.

Ethical approval All procedures performed in studies involving human participants were in accordance with the 1964 Helsinki declaration and its later amendments or comparable ethical standards. The study was performed as a part of a service evaluation study and formal ethical review was, therefore, not required as part of our local institutions standards.

Informed consent Informed consent was obtained from all individual participants included in the study.

Open Access This article is distributed under the terms of the Creative Commons Attribution 4.0 International License (http://creativeco mmons.org/licenses/by/4.0/), which permits unrestricted use, distribution, and reproduction in any medium, provided you give appropriate credit to the original author(s) and the source, provide a link to the Creative Commons license, and indicate if changes were made. 


\section{References}

1. Katsoulis E, Court-Brown C, Giannoudis PV. Incidence and aetiology of anterior knee pain after intramedullary nailing of the femur and tibia. J Bone Jt Surg Br. 2006;88:576-80 (PMID: 16645100).

2. Ostrum RF, Agarwal A, Lakatos R, Poka A. Prospective comparison of retrograde and antegrade femoral intramedullary nailing. $J$ Orthop Trauma. 2000;14:496-501.

3. Toms AD, Morgan-Jones RL, Spencer-Jones R. Intramedullary femoral nailing: removing the nail improves subjective outcome. Injury. 2002;33:247-9.

4. Courtney PM, Boniello A, Donegan D, Ahn J, Mehta S. Functional outcomes in infrapatellar and suprapatellar tibial nailing: does approach matter? Am J Orthop. 2015;44:13-6 (PMID: 26665254).

5. Chan DS, Serrano-Riera R, Griffing R, Stevenson B, Infrante A, Watson D, Sagi HC, Sanders RW. Suprapatellar versus infrapatellar tibial nail insertion: a prospective randomized control pilot study. J Orthop Trauma. 2016;30(3):130-4.

6. Patt JC, Mauerhan DR. Outcomes research in total joint replacement: a critical review and commentary. Am J Orthop (Belle Mead NJ). 2005;34:167-72 (PMID: 15913169).

7. Davies AP. Rating systems for total knee replacement. Knee. 2002;9:261-6 (PMID: 12424032).

8. Kujala UM, Jaakkola LH, Koskinen SK, Taimela S, Hurme M, Nelimarkka O. Scoring of patellofemoral disorders. Arthroscopy. 1993;9:159-63 (PMID: 8461073).

9. Fulkerson JP, Becker GJ, Meaney JA, Miranda M, Folcik MA. Anteromedial tibial tubercle transfer without bone graft. Am J Sports Med. 1990;18:490-6 (PMID: 2252090).

10. Konan S, Hossain F, Patel S, Haddad FS. Measuring Function after hip and knee surgery: the evidence to support performancebased functional outcome tasks. Bone Jt J. 2014;96:1431-5 (PMID: 25371452).
11. Konan S, Tahmassebi J, Haddad FS. The development and validation of a more discriminating functional hip score for research. HSS J. 2012;8:198-205.

12. Wamper KE, Sierevelt IN, Poolman RW, Bhandari M, Haverkamp D. The Harris hip score: do ceiling effects limit its usefulness in orthopedics? Acta Orthop. 2010;81:703-7.

13. Souer JS, Lozano-Calderon SA, Ring D. Predictors of wrist function and health status after operative treatment of fractures of the distal radius. J Hand Surg Am. 2008;33:157-63 (PMID: 18294534).

14. Rosenberger PH, Jokl P, Ickovics J. Psychosocial factors and surgical outcomes: an evidence-based literature review. J Am Acad Orthop Surg. 2006;14:397-405 (PMID: 16822887).

15. Podsialdo D, Richardson S. The timed "Up \& Go": a test of basic function for frail elderly persons. J Am Geriatr Soc. 1991;39:142-8 (PMID: 1991946).

16. Steffen TM, Hacker TA, Mollinger I. Age and gender related test performance in community-dwelling elderly people: Six-minute Walk Test, Berg Balance Scale, Timed Up \& Go Test, and gait speeds. Phys Ther. 2002;82:128-37 (PMID: 11856064).

17. Rejeski WJ, Ettinger WH Jr, Schummaker S, James P, Burns R, Elam JT. Assessing performance related disability in patients with knee osteoarthritis. Osteoarthr Cartil. 1995;3:157-67 (PMID: 8581745).

18. Bade MJ, Wolfe P, Zeni JA, Stevens-Lapsley JE, Snyder-Mackler L. Predicting poor physical performance after total knee arthroplasty. J Orthop Res. 2012;30:1805-10 (PMID: 22539338).

19. Irrgang JJ, Snyder-Mackler L, Wainner RS, Fu FH, Harner CD. Development of a patient-reported measure of function of the knee. J Bone Jt Surg Am. 1998;80:1132-45 (PMID: 9730122).

20. Barker SL, Rehman H, McCullough AL, Fielding S, Johnstone AJ. Assessment following distal radius fractures: a comparison of 4 scoring systems, visual numerical scales and objective measurements. J Hand Surg Am. 2016;41:219-24 (PMID: 26684714). 